\title{
CMS Outer Tracker Upgrade Plans
}

\author{
Doris Eckstein ${ }^{* \dagger}$ \\ Deutsches Elektronen-Synchrotron DESY, Hamburg \\ E-mail: doris.ecksteinedesy.de
}

\begin{abstract}
With the upgrade of the LHC to the high-luminosity LHC (HL-LHC), the tracker system of the CMS experiment will have to be upgraded. It is planned to use information from the tracker in the first level trigger of CMS. For this purpose CMS plans to introduce $\mathrm{p}_{\mathrm{T}}$ modules which will provide trigger capability. The luminosity increase by a factor of five in the HL-LHC compared to the design luminosity will lead to higher occupancy and radiation levels in the sensors. In order to cope with these, the strip granularity will be significantly increased and the sensors of the upgraded tracker will have to be cooled to a temperature of $-20^{\circ} \mathrm{C}$. Moreover, the sensor material and design have to be chosen carefully in order to withstand the anticipated radiation. This paper introduces the concept of the $\mathrm{p}_{\mathrm{T}}$ module and gives an overview of the ongoing $\mathrm{R} \& \mathrm{D}$ activities concerning the silicon sensor material.
\end{abstract}

22nd International Workshop on Vertex Detectors (Vertex 2013)

September 15-20, 2013

Lake Starnberg, Germany

*Speaker

${ }^{\dagger}$ for the CMS Tracker Collaboration 


\section{Introduction}

The large Hadron Collider (LHC) at CERN delivered about $30 \mathrm{fb}^{-1}$ of proton-proton collision data at center-of-mass energies of 7 and $8 \mathrm{TeV}$ to CMS until the end of Run 1 in 2012. In order to enhance the discovery potential of the accelerator, several luminosity upgrades are foreseen in the next ten years. Currently, accelerator and experiments are preparing for Run 2 at $14 \mathrm{TeV}$ which will start in 2015 and is expected to reach a peak luminosity of $1.7 \cdot 10^{34} \mathrm{~cm}^{-2} \mathrm{~s}^{-1}$ lasting until Long Shutdown 2 in 2018. Improvements especially to the injector chain will allow to reach luminosities of $2 \cdot 10^{34} \mathrm{~cm}^{-2} \mathrm{~s}^{-1}$ until in Long Shutdown 3 the ultimate stage, the High Luminosity LHC (HL-LHC) will be reached. With a peak luminosity of $5 \cdot 10^{34} \mathrm{~cm}^{-2} \mathrm{~s}^{-1}$ and an expected average of 140 collisions per bunch-crossing in CMS, the HL-LHC will commence operations in 2025 and is expected to deliver $3000 \mathrm{fb}^{-1}$ of data, thus presenting an extremely challenging environment to the experiments. Several components of the CMS experiment [1] will be upgraded in order to cope with these conditions while retaining the performance of the current detector.

\section{The Phase-2 Outer Tracker Upgrade}

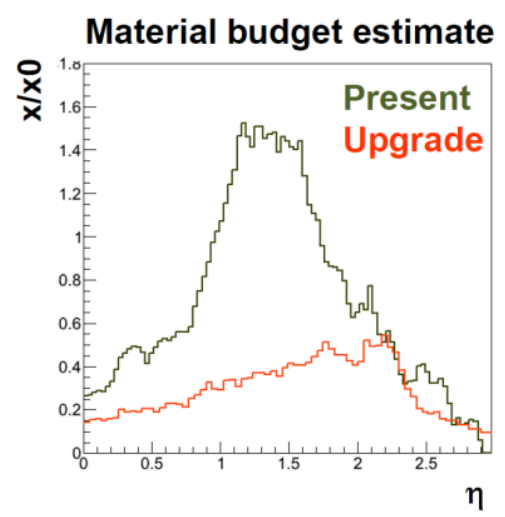

Figure 1: Material budget in units of radiation length $\mathrm{X}_{0}$ of the current CMS tracker and the current extimate for the upgraded tracker.

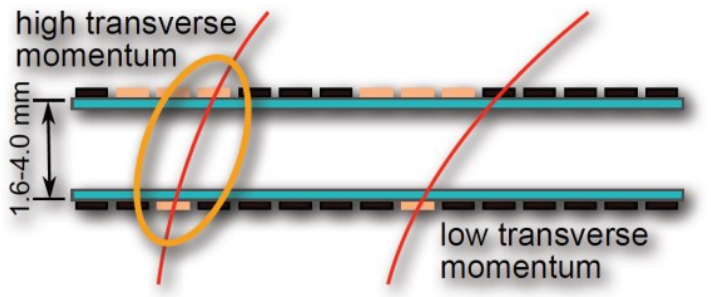

Figure 2: Illustration of the Track Trigger concept of correlated hits in closely spaced sensors. Tracks bend in the CMS magnetic field and clusters in both sensors are correlated to distinguish high and low $\mathrm{p}_{\mathrm{T}}$.

The main requirements for the upgraded tracker arise from the conditions at the HL-LHC. The new detector must be radiation tolerant, in order to be able to operate efficiently up to the envisaged integrated luminosity of $3000 \mathrm{fb}^{-1}$ without the possibility of exchanging components of the tracker. An increased granularity will ensure strip occupancy of not more than a few percent and allow an efficient tracking performance at pileup of 100-200. The aim is to reduce material in the tracking volume with respect to the present tracker (Figure 1), in order to improve the tracking performance at low $\mathrm{p}_{\mathrm{T}}$ and reduce the rate of secondary interactions. Finally, because of the increased rate and pileup, the event selection would become very challenging. Therefore, tracking information is required to provide input to the Level 1 (L1) 
trigger of CMS in order to reduce the trigger rate and preserve the physics performance of CMS.

\subsection{Concept and Layout}

The concept of the upgraded tracker is based on the " $\mathrm{p}_{\mathrm{T}}$-module", illustrated in Figure 2. The module is composed of two parallel sensors which are separated by 1.6 or $4 \mathrm{~mm}$ depending on the position in the detector. Both sensors are read out by the same front-end chip. This allows discriminating high- $\mathrm{p}_{\mathrm{T}}$ from low- $\mathrm{p}_{\mathrm{T}}$ tracks based on the bending in the $3.8 \mathrm{~T}$ magnetic field of the CMS solenoid and by selecting hits within a search window. "Stubs" are formed from the selected correlated clusters in the top and the bottom sensor.

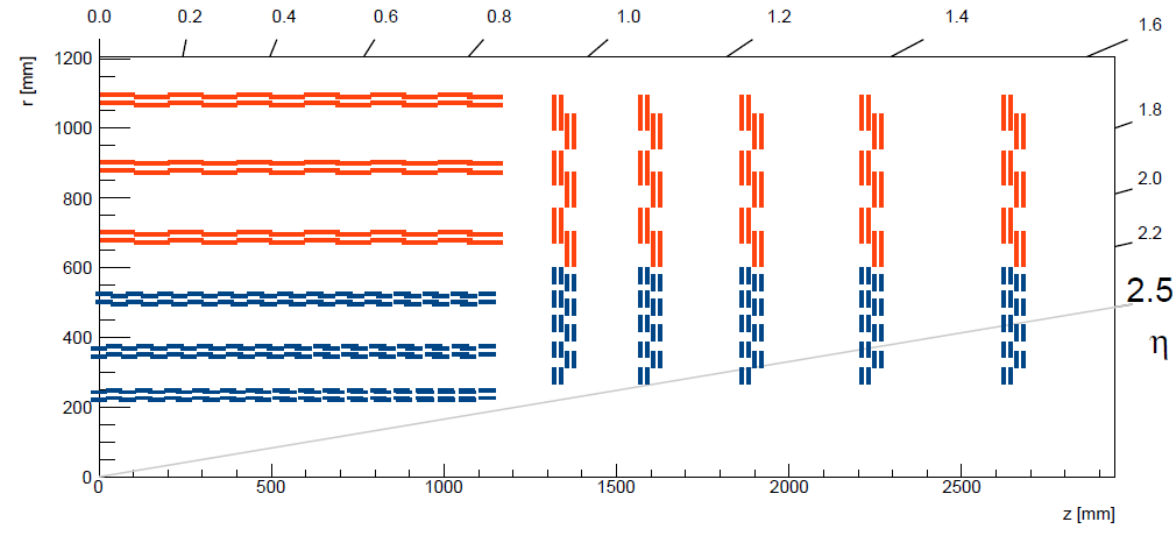

Figure 3: Baseline layout of the CMS Tracker for the HL-LHC. Each line presents one $\mathrm{p}_{\mathrm{T}}$ module, blue the PS and red the $2 \mathrm{~S}$ type (see text).

Figure 3 shows the baseline layout with six layers in a barrel and five disks in each end cap. It was chosen after detailed studies with a tool developed within CMS, tkLayout [2], that evaluates the performance of given tracker layouts using analytical estimates of parameters such as for example stub finding efficiency and occupancy.

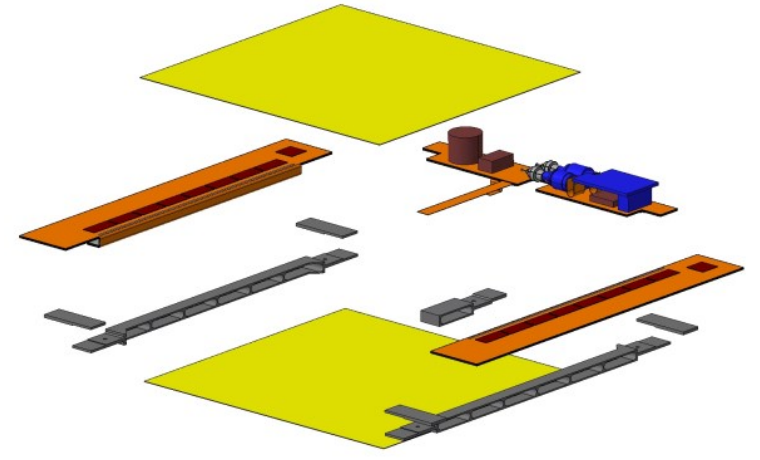

(a) $2 \mathrm{~S}$ module

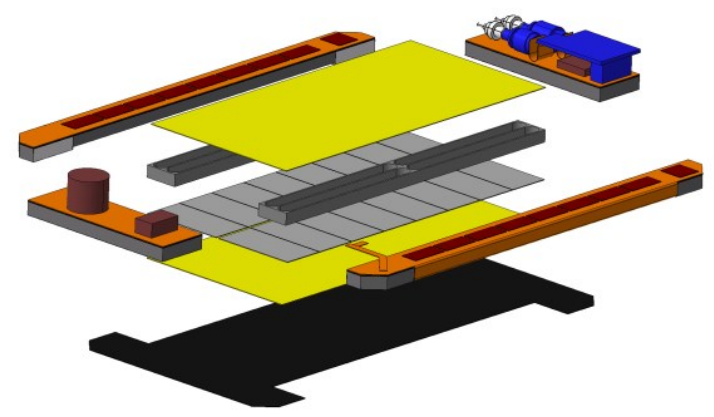

(b) PS module

Figure 4: Exploded view of the $2 \mathrm{~S}$ and the PS module: sensors (yellow), hybrids (orange), $2 \times 8$ front-end chips and the concentrators (red), MPA (in light grey), GBT chip and opto-electronic connector (blue), DCDC converter (brown cylinder shape), Al-CF bridge (grey). 
Two types of modules are being developed for installation in different parts of the CMS tracker, as depicted in Figure 4. The $2 \mathrm{~S}$ module uses two identical silicon sensors with an area of $10 \mathrm{~cm} \mathrm{x} 10 \mathrm{~cm}$, a strip length of $5 \mathrm{~cm}$ and $90 \mu \mathrm{m}$ pitch. It is to be installed in the outer part of the tracker. The PS module uses one strip sensor with $2.5 \mathrm{~cm}$ strip length and $100 \mu \mathrm{m}$ pitch, and one pixel sensor with $1.5 \mathrm{~mm}$ long and $100 \mu \mathrm{m}$ wide pixels, both with an area of $5 \mathrm{~cm} \mathrm{x} 10 \mathrm{~cm}$. It will be used in the inner part of the tracker at radii below $60 \mathrm{~cm}$. Here, the track density is higher and the PS module will measure the position of the stubs with a resolution of $O(1) \mathrm{mm}$ in beam direction and thus help to identify the primary vertex. The placement of the $2 \mathrm{~S}$ and PS modules in the tracker layout can be seen in Figure 3. As depicted in Figure 4, both modules integrate on the dedicated service hybrid a DC-DC converter [3], and a GigaBitTransceiver (GBT) chip and opto-electronic connector [4].

In the $2 \mathrm{~S}$ module, both strip sensors will be wire-bonded to the hybrid board onto which 8 CMC Binary Chips (CBC) [5] per side are connected with C4 bump bonds. The chip is implemented in a binary, unsparsified architecture. Each chip receives data from 127 channels of both the top and the bottom sensor, performs signal amplification, hit detection and cluster finding. It also hosts the stub finding logic, in which clusters from the top and the bottom sensor are correlated using a programmable matching window of up to \pm 8 channels, programmable offsets to compensate for mis-alignments and an also programmable cluster width. Per bunch crossing up to three stubs can be read out and passed to the concentrator chip, which will pack the data from $8 \mathrm{CBCs}$ and send up to 12 stubs per 8 bunch crossings to the GigaBitTransceiver.

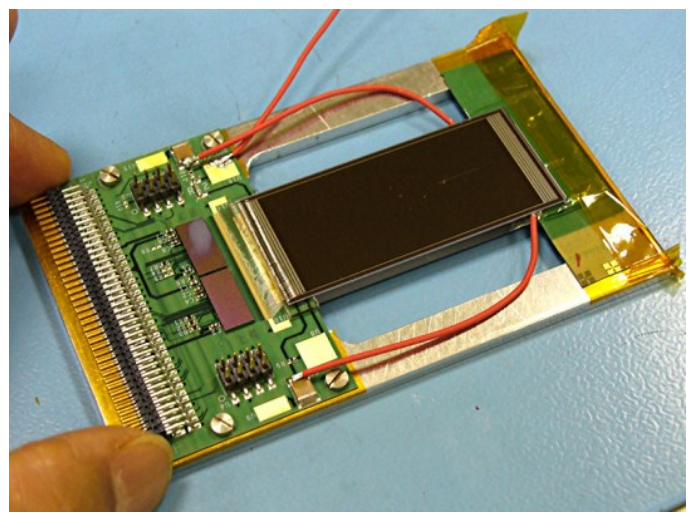

Figure 5: Photo of a $2 \mathrm{CBC} 2$ module.

Recently, tests with the second prototype version of the $\mathrm{CBC}$ chip, the $\mathrm{CBC} 2$, were performed on a mini- p $_{\mathrm{T}}$-module hosting two $\mathrm{CBC} 2$ chips and two strip sensors (Figure 5). This demonstrator was used in order to evaluate the chip functionality in laboratory tests with a source and with cosmic rays and it has shown that stubs are correctly found. Testbeam measurements with these mini-modules were carried out at the end of 2013 to demonstrate the trigger capability.

In the PS module, the strip sensor will be read out by the Short-Strip-Asic (SSA) and the pixel sensor will be bonded to the Macro Pixel ASIC (MPA) [6]. The MPA will also collect 
data from the SSA and perform the stub finding. It is currently being designed in CMOS $65 \mathrm{~nm}$ technology.

\subsection{Module Mechanics and Cooling}

The design of the modules concentrated so far on optimizing the $2 \mathrm{~S}$ module, also in terms of mechanics and cooling. The experience with this serves as a good basis for the PS one. A big effort went into designing and simulating the $2 \mathrm{~S}$ module. The boundary conditions are defined through the HL-LHC radiation environment as simulated by FLUKA calculations [7] and the precision and stability required by the trigger capability of the modules. The high irradiation levels require the sensors to be operated at a stable temperature of $-20^{\circ} \mathrm{C}$. Two-phase $\mathrm{CO}_{2}$ cooling is the technology chosen for the whole tracker. When optimizing the design of the mechanical supports, possible deformations and stress due to coefficient of thermal expansion (CTE) mismatch have to be taken into account. The optimizations were supported by Finite Element (FE) calculations.

For the $2 \mathrm{~S}$ module mechanics, the optimizations have led to a design using a hollow unibody bridge made of carbon-fiber reinforced aluminum (Al-CF) composite material, see also Figure 4. The CTE of Al-CF matches nicely the one of silicon, it has a high thermal conductivity and adds little to the material budget.

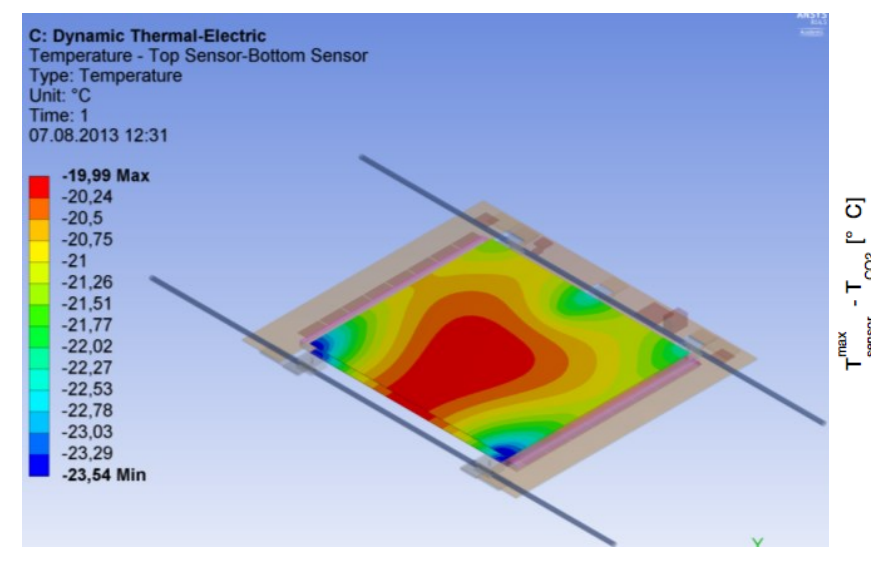

(a) Temperature distribution on the sensors for a $4 \mathrm{~mm}$ $2 \mathrm{~S}$ module. $\mathrm{CO}_{2}$ coolant temperature is at $-28.6^{\circ} \mathrm{C}$.

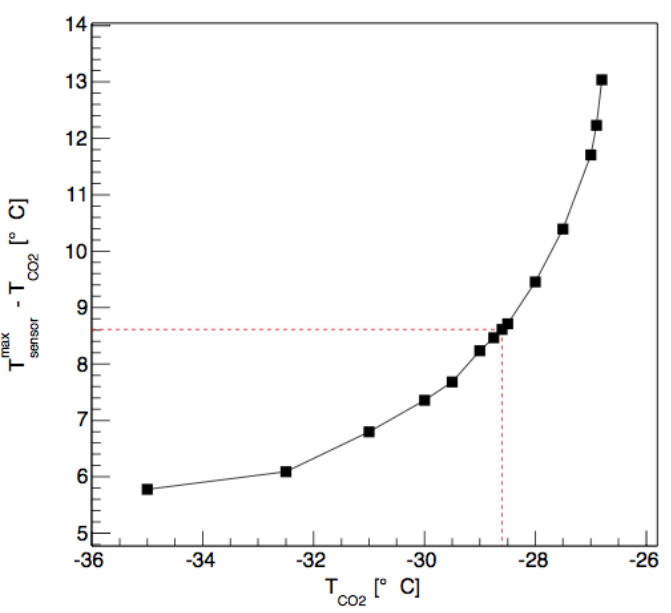

(b) Temperature difference between sensor (maximum) and $\mathrm{CO}_{2}$ versus $\mathrm{CO}_{2}$ temperature. The red lines indicate the working point of $-20^{\circ} \mathrm{C}$ sensor temperature.

Figure 6: Results from thermal Finite Element simulation for a $2 \mathrm{~S}$ module.

Finite Element calculations have been performed in order to support the design optimization regarding mechanical stability and regarding the thermal performance. Figure 7a shows the temperature distribution on both sensors of a $2 \mathrm{~S}$ module with $4 \mathrm{~mm}$ sensor spacing. In this simulation the temperature of the $\mathrm{CO}_{2}$ was tuned to obtain a maximum of $-20^{\circ} \mathrm{C}$ on the sensors, and the heat loads were taken into account as follows: $200 \mathrm{~mW}$ per concentrator, $1 \mathrm{~W}$ for the DC-DC converter, $800 \mathrm{~mW}$ for the optical transmitter, $1.22 \mathrm{~W}$ for $2 \mathrm{x} 8 \mathrm{CBC}$ chips. The 
sensor power dissipation was calculated for a fluence of $7.35 \cdot 10^{14} \mathrm{n}_{\mathrm{eq}} / \mathrm{cm}^{2}$ for $200 \mu \mathrm{m}$ thick sensors at $600 \mathrm{~V}$ bias. The temperature gradient on the sensors is $3.5^{\circ} \mathrm{C}$ and the $\mathrm{CO}_{2}$ has to be kept at $-28.6^{\circ} \mathrm{C}$ in order to obtain $-20^{\circ} \mathrm{C}$ on the sensors. In Figure $6 \mathrm{~b}$ the difference of the sensor and the $\mathrm{CO}_{2}$ temperatures is plotted as function of the $\mathrm{CO}_{2}$ temperature. The red lines mark the point, where the maximum sensor temperature is at $-20^{\circ} \mathrm{C}$. Increasing the $\mathrm{CO}_{2}$ temperature would result in a higher sensor temperature and higher sensor power dissipation until at a $\mathrm{CO}_{2}$ temperature of $-27^{\circ} \mathrm{C}$ thermal runaway would occur.

\subsection{Sensor development for the CMS Outer Tracker}

\subsubsection{Materials and Irradiations}

An R\&D campaign was initiated within the CMS collaboration in order to identify the sensor material suitable for the upgraded tracker. This campaign aimed at comparing a variety of silicon materials produced with different growth techniques processed under similar conditions with the same wafer layout in order to exclude possible influences of the processing at different sensor manufacturers. Therefore, all sensors were produced by Hamamatsu Photonics K.K.

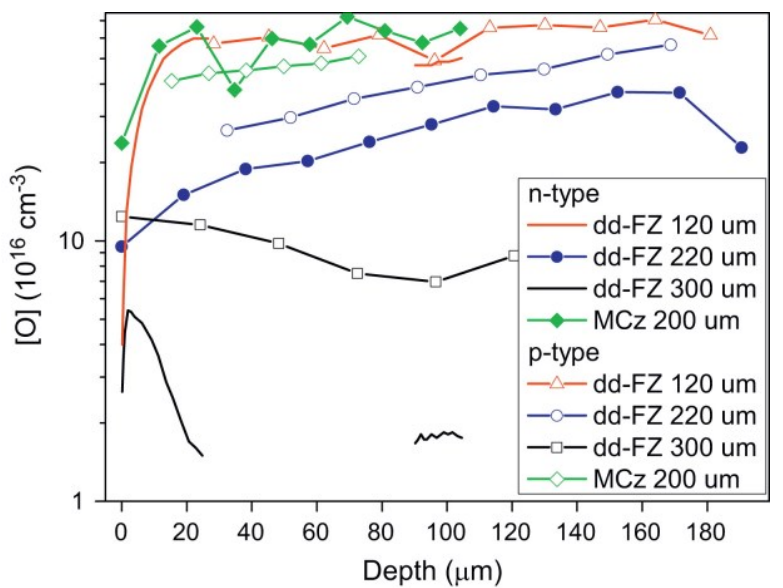

Figure 7: Oxygen concentration measured by Secondary Ion Mass Spectroscopy (SIMS) for sensors from $\mathrm{MCz}$ and dd-FZ material of different thickness. Continuous lines denote a first measurement set, lines with symbols mark the second set.

Results of previous R\&D within e.g. the RD50 Collaboration ${ }^{1}$ have shown that the charge trapping as well as the leakage current increase with the fluence independent of the silicon material chosen. In contrast, the change of the effective doping concentration $\mathrm{N}_{\text {eff }}$ will depend on the material and it will change the electric field distribution in the device. The main parameter influencing this change is the amount of oxygen which is introduced during either the growth process or the sensor processing. The materials used in the CMS R\&D campaign are $200 \mu \mathrm{m}$ thick Magnetic Czochralski (MCz), standard Float Zone (FZ) silicon of $200 \mu \mathrm{m}$, and deep-diffused FZ (dd-FZ) of $100 \mu \mathrm{m}, 200 \mu \mathrm{m}$ and $300 \mu \mathrm{m}$ thickness. The active thicknesses of $100 \mu \mathrm{m}$ and $200 \mu \mathrm{m}$, which are smaller than the wafer thickness of $320 \mu \mathrm{m}$ were achieved in the

\footnotetext{
${ }^{1}$ rd50.web.cern.ch
} 
dd-FZ material through a high-temperature deep-diffusion of dopants from the rear sensor side. In this material oxygen concentration levels were measured which are much higher than expected for standard FZ material, as shown in Figure 7. This was found to be related to the high-temperature treatment in the deep-diffusion process and the connected diffusion of oxygen from the rear side surface layer into the bulk [8].

Sensors were produced in p-in-n as well as in n-in-p technology using p-spray and p-stop isolation techniques for the latter. A variety of test structures were implemented, for example planar diodes and mini-strip and pixel sensors in order to investigate the electrical and charge collection properties before and after irradiation. A mixed particle irradiation scenario was used in order to mimic the irradiation levels and composition in the Outer Tracker region of the HLLHC. Therefore, the sensors were first irradiated with either protons or neutrons, characterized and then irradiated with the other particle type before the cumulative damage was characterized. For a subset of sensors, especially the planar diodes, $60^{\circ} \mathrm{C}$ and $80^{\circ} \mathrm{C}$ annealing and subsequent measurements were performed also after single particle irradiation. This provides a comprehensive set of information of the single particle and mixed irradiations. Reactor neutron $(\kappa=0.91)$ irradiations have been carried out at the TRIGA Mark II reactor in Ljubljana, Slovenia ${ }^{1}$. Proton irradiations have been obtained from two facilities providing protons with different energies: $23 \mathrm{MeV}$ protons $(\kappa=2.00)$ from ZAG, Karlsruhe, Germany ${ }^{2}$, and $23 \mathrm{GeV}$ protons $(\kappa=0.62)$ from the Proton Synchrotron PS at CERN ${ }^{3}$.

\subsubsection{Measurement results}

Pad diodes have been used to study the evolution of the full depletion voltage $V_{\text {dep }}$ extracted from Capacitance-Voltage measurements and, correspondingly, the effective doping concentration $\left(\mathrm{N}_{\mathrm{eff}}\right)$ after irradiation. One important result of this $\mathrm{R} \& \mathrm{D}$ campaign is summarized in Figure 8. It presents the absolute value of $\mathrm{N}_{\text {eff }}$ and $\mathrm{V}_{\text {dep }}$ as they develop with irradiation fluence for $\mathrm{FZ}, \mathrm{MCz}$ and dd-FZ materials. In the irradiation with $23 \mathrm{GeV}$ protons, illustrated in Figure 8a, a smaller introduction rate for $\mathrm{FZ}$ than for $\mathrm{MCz}$ can be observed. Annealing behavior and Transient Current Technique measurements show that the p-type $\mathrm{MCz}$ as well as the n-type FZ materials undergo "type inversion", while the n-type MCz and the p-type FZ not ${ }^{4}$. After the irradiation with $23 \mathrm{MeV}$ protons, Figure 8b, the situation looks different: the n-type $\mathrm{MCz}$ as well as the n-type (oxygen-rich) dd-FZ have undergone "type-inversion", while the p-type sensors of both flavors have not. Those sensors which do not type-invert end up at a higher depletion voltage at high fluences. In summary we conclude that high oxygen content makes silicon of both polarities more sensitive to the type and energy of the irradiating particle. This has been observed previously by the LHCb VELO [9], whose p-type layer is made of diffusionoxygenated FZ and shows a steeper than expected increase in $\mathrm{V}_{\text {dep }}$ with fluence. In order to investigate the proton energy dependence, CMS has conducted irradiation in Los Alamos with

\footnotetext{
${ }^{1}$ http://www.rcp.ijs.si/ric/index-a.htm

${ }^{2} \mathrm{http}: / /$ www.zyklotron-ag.de

${ }^{3} \mathrm{http}: / /$ irradiation.web.cern.ch/irradiation/irrad1.htm

${ }^{4}$ The term ,type inversion“ is used here to summarize the change in the electric field distribution and the effect, that the effective junction is moving from the front to the rear sensor side. However, the electric field distribution is much more complicated (double junction effect).
} 
proton energies of $800 \mathrm{MeV}$, i.e. in the range between the CERN PS and the ZAG Karlsruhe. This energy, according to simulations [7], will correspond to an energy in the peak region of charged hadrons in CMS at the HL-LHC.

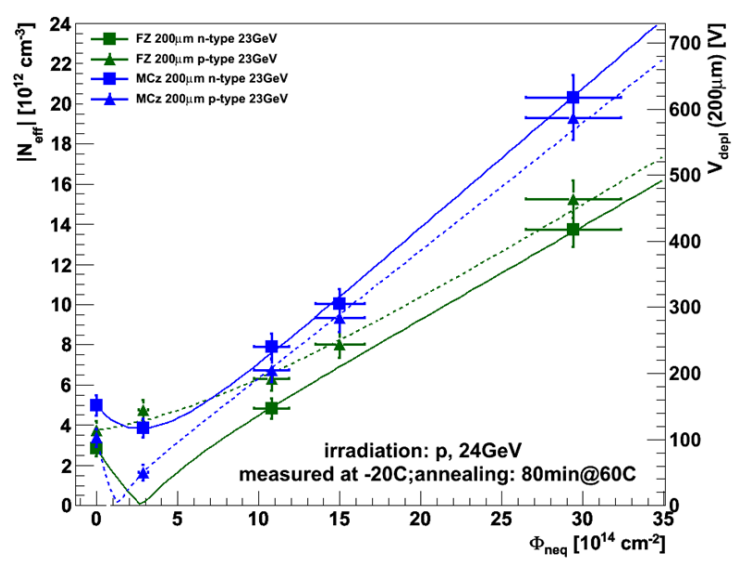

(a) The change of $\mathrm{N}_{\text {eff }}$ in $\mathrm{FZ}$ and $\mathrm{MCz}$ sensors after $23 \mathrm{GeV}$ proton irradiation.

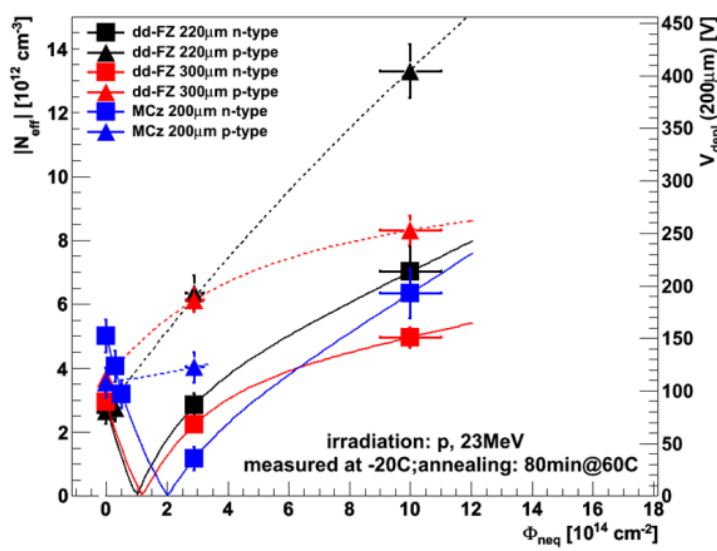

(b) The change of $\mathrm{N}_{\text {eff }}$ in dd-FZ and $\mathrm{MCz}$ sensors after $23 \mathrm{MeV}$ proton irradiation.

Figure 8: $\mathrm{N}_{\text {eff }}$ and $\mathrm{V}_{\text {dep }}$ scaled to $200 \mu \mathrm{m}$ of silicon for sensors after irradiation with protons. The diode sensors were annealed for 80 minutes at $60^{\circ} \mathrm{C}$. The lines indicate the fit through the data points, dashed the p-type sensors, continuous lines the n-type sensors.

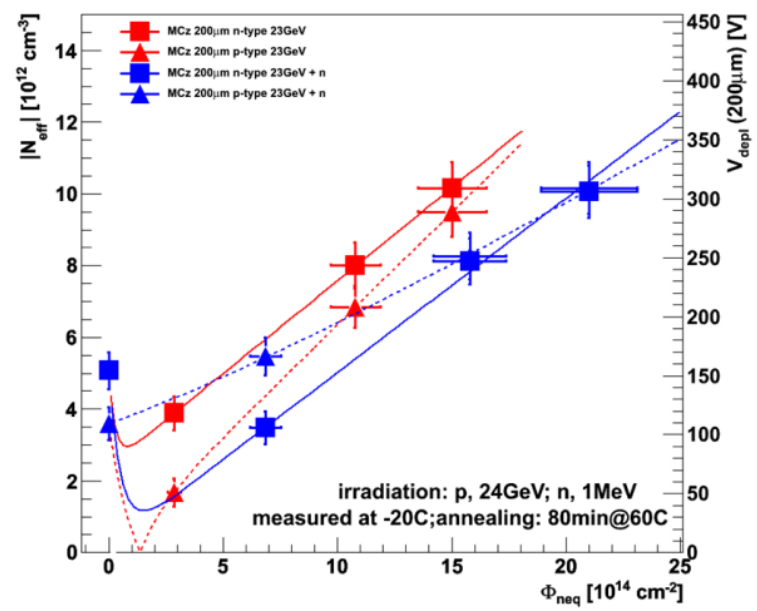

Figure 9: The change of $\mathrm{N}_{\text {eff }}$ and $\mathrm{V}_{\text {dep }}$ in $\mathrm{MCz}$-and p-type sensors after $23 \mathrm{GeV}$ proton irradiation. The red symbols show proton only and the blue the mixed irradiations; annealing is $80 \mathrm{~min}$ at $60^{\circ} \mathrm{C}$.

As mentioned before, mixed irradiations have been performed in order to study compensation effects as observed first by Kramberger et al. [10]. A summary is shown in Figure 9 of the $\mathrm{N}_{\text {eff }}$ and $\mathrm{V}_{\text {dep }}$ as a function of fluence for $\mathrm{MCz}$ material. The plot shows in red the sensors which received a pure $23 \mathrm{GeV}$ proton irradiation with fluences of $3 \cdot 10^{14}, 1 \cdot 10^{15}$ and $1.5 \cdot 10^{15} \mathrm{n}_{\mathrm{eq}} / \mathrm{cm}^{2}$. The blue symbols depict the results of the mixed irradiations after neutron fluences of $4 \cdot 10^{14}, 5 \cdot 10^{14}$ and $6 \cdot 10^{14} \mathrm{n}_{\mathrm{eq}} / \mathrm{cm}^{2}$ were added respectively. For the n-type MCz (box 
symbols and solid lines) clearly the $\mathrm{V}_{\text {dep }}$ is smaller in case of the mixed compared to the pure proton irradiation. For the p-type $\mathrm{MCz}$ (triangles and dashed lines), a much steeper rise of $\mathrm{V}_{\text {dep }}$ is observed in the fluence region beyond the "type inversion" of the purely proton irradiated sample compared to the sample with proton plus neutron irradiation.

In order to select the material for the upgraded Outer Tracker the charge collection was one of the major aspects of the investigations. Dedicated mini-strip sensors were tested in laboratory setups with radioactive sources and in testbeams. As the CBC readout chip for the upgraded Outer Tracker will be a binary chip, the figure of merit is that the seed signal exceeds a minimum threshold. Figure 10 summarizes the annealing of the signal in the seed strip of different sensor types biased to $600 \mathrm{~V}$ after mixed fluences of $7 \cdot 10^{14} \mathrm{n}_{\mathrm{eq}} / \mathrm{cm}^{2}$ and $1.5 \cdot 10^{15} \mathrm{n}_{\mathrm{eq}} / \mathrm{cm}^{2}$. It is clear from both plots that while the $200 \mu \mathrm{m}$ sensors have a flat annealing behavior, the signal in the $300 \mu \mathrm{m} \mathrm{FZ} \mathrm{p-in-n} \mathrm{sensor} \mathrm{drops} \mathrm{rather} \mathrm{quickly} \mathrm{and} \mathrm{the} 300 \mu \mathrm{m} \mathrm{FZ} \mathrm{n-in-p} \mathrm{sensor}$ shows a beneficial annealing before dropping down due to under-depletion. Please note that at large fluences above $7 \cdot 10^{14} \mathrm{n}_{\mathrm{eq}} / \mathrm{cm}^{2}$ in the $\mathrm{p}$-in-n sensors the signal was not anymore distinguishable from the Random Ghost Hits which appeared [11]. These hits are not correlated with the signal but originate from random noise distributed over the whole sensor.

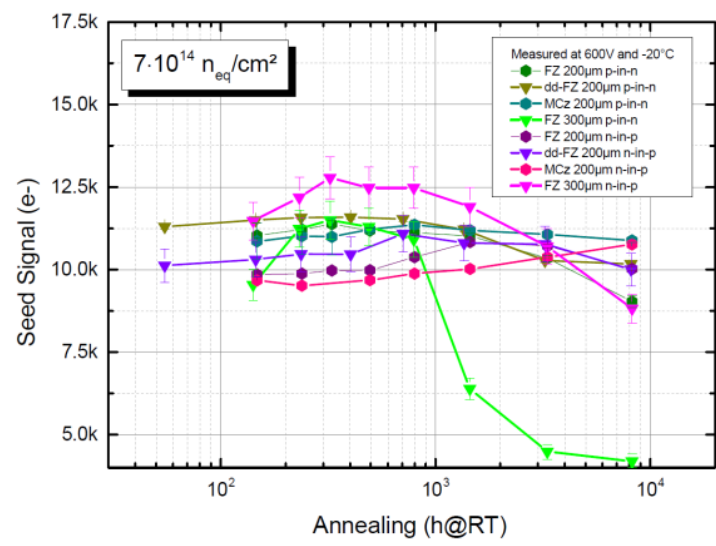

(a) 200 and $300 \mu \mathrm{m}$ thick sensors irradiated to $7 \cdot 10^{14} \mathrm{n}_{\mathrm{eq}} / \mathrm{cm}^{2}$.

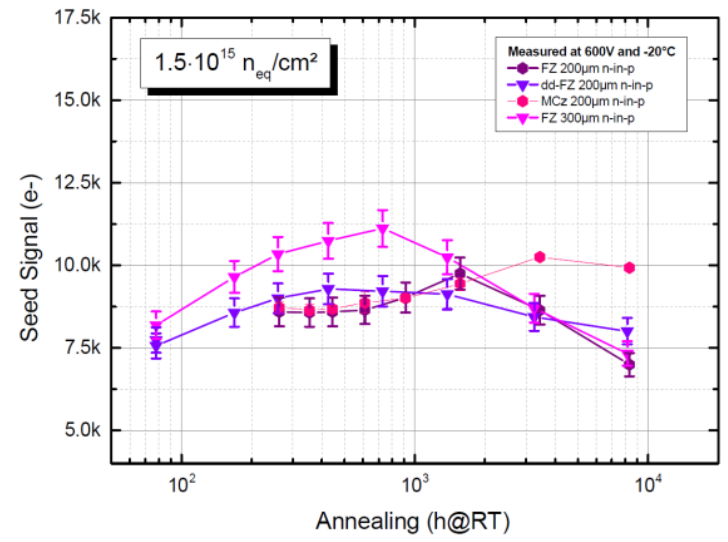

(b) $200 \mu \mathrm{m}$ thick sensors irradiated to $1.5 \cdot 10^{15}$ $\mathrm{n}_{\mathrm{eq}} / \mathrm{cm}^{2}$.

Figure 10: Annealing of the seed signal of FZ, dd-FZ and $\mathrm{MCz}$ sensors. Sensors are biased to $600 \mathrm{~V}$ and cooled to $-20^{\circ} \mathrm{C}$. Annealing was scaled to room temperature $\left(21^{\circ} \mathrm{C}\right)$.

The information extracted from the CMS sensor R\&D campaign, explicitly the appearance of the Random Ghost Hits in the p-in-n sensors and the flat annealing behaviour led to the decision to use n-in-p sensors in the upgraded CMS Outer Tracker. The current baseline concerning sensor thickness is $200 \mu \mathrm{m}$ with a bias voltage of $600 \mathrm{~V}$ because the annealing behaviour as it is summarized in Figure 10 gives a nice handle to control the power consumption of the sensor. Now, the CMS sensor development for the Phase 2 Outer Tracker will concentrate on working out a recommendation concerning the oxygen level, the optimization of the sensor layout and on evaluating possible vendors. 


\subsection{Summary}

The Phase 2 upgrade of the CMS Outer Tracker is progressing well. A baseline layout for the tracker has been chosen and the design of the $\mathrm{p}_{\mathrm{T}}$ modules is taking shape, with the $2 \mathrm{~S}$ module being well advanced. Further optimizations of these modules are under evaluation and provide input to further developments of prototypes. A CMS campaign to evaluate silicon sensor material for the upgraded tracker has provided interesting results and has led to the choice of p-bulk material as baseline. Further steps now are the optimization of the sensor design and the qualification of possible vendors.

\section{Acknowledgements}

The research leading to these results has received funding from the European Commission under the FP7 Research Infrastructures project AIDA, grant agreement no. 262025. The information herein only reflects the views of its authors and not those of the European Commission and no warranty expressed or implied is made with regard to such information or its use.

\section{References}

[1] CMS Collaboration, The CMS experiment at the CERN LHC, 2008 JINST 3 S08004.

[2] S. Mersi et al., Software package for the characterization of Tracker layouts, $13^{\text {th }}$ ICATPP 2011, World Scientific, Vol.7 of the Astroparticle, Particle, Space Physics, Radiation Interaction, Detectors and Medical Physics Application Series (2012).

[3] L. Feld, Novel powering schemes for pixel and tracking detectors, this issue.

[4] L. Amaral et al., The versatile link, a common project for super-LHC, 2009 JINST 4 P12003.

[5] D. Braga et al., CBC2: a microstrip readout ASIC with coincidence logic for trigger primitives at HL-LHC, 2012 JINST 7 C10003.

[6] A. Marchioro, A hybrid module architecture for a prompt momentum discriminating tracker at SLHC, 2011 PoS(Vertex 2011)037.

[7] S. Müller, The Beam Condition Monitor 2 and the Radiation Environment of the CMS Detector at the HL-LHC, 2010, CMS-TS-2010-042.

[8] A. Junkes, Influence of radiation induced defect clusters on silicon particle detectors, 2011, DESY-THESIS-2011-031

[9] A. Affolder et al., Radiation damage in the LHCb vertex locator, 2013 JINST 8 P08002

[10]G. Kramberger et al., NIM A609 (2009), 142-148.

[11]A. Dierlamm, Planar sensors for future vertex and tracking detectors, this issue. 